\title{
ON THE MASS DISCRETIZATION APPROACH TO ELASTODYNAMICS OF PERIODICALLY STRATIFIED COMPOSITES
}

\author{
Olga Szlachetka ${ }^{1}$, Monika Wagrowska ${ }^{1}$, Joanna Witkowska-Dobrev ${ }^{1}$, Czestaw Woźniak ${ }^{2}$ \\ ${ }^{I}$ Faculty of Civil and Environmental Engineering, Warsaw University of Life Sciences \\ Warszawa, Poland \\ ${ }^{2}$ Faculty of Civil Engineering, Architecture and Environmental Engineering \\ Technical University of Lodz, Lódź, Poland \\ olga_szlachetka@sggw.pl,monika_wagrowska@sggw.pl
}

\begin{abstract}
The object of analysis are two component periodically stratified linear elastic multilayered composites. The aim of contribution is elastodynamics of these composites subjected to the mass discretization on the interfaces between adjacent homogeneous layers. Two specific cases of the mass discretizations are taken into account. In the first case the mass is assigned uniformly to every interface. It is shown that this kind of mass discretization leads to physically completely wrong results. In the second case, the mass is discretized uniformly but on the every second interface. In this case it is shown that the obtained elastodynamics equations have a physical sense provided that the wave are sufficiently long.
\end{abstract}

\section{Introduction}

It is known that in elastodynamics of continuous nonhomogeneous media the direct approach to the analysis of specific problems is very complicated. We can mention here, e.g., the wave propagation problems in periodically stratified composites. To be more exact the wave propagation problems can be properly modelled by the mass discretization provided that the waves are sufficiently long. It means that the wavelengths are large when compared to the microstructure length of periodically spaced elastic medium. In the subsequent Section for the sake of simplicity we shall specified the object of analysis as a two component periodically stratified linear elastic space. It is assumed that: the material space under consideration is unbounded, linear elastic and made of two isotropic components which are periodically distributed in one direction. Hence the interfaces between adjacent materials are represented by the infinite system of parallel planes. Moreover, the homogeneous layers of the medium are assumed to have the same thickness. The analysis will be restricted exclusively to the waves propagating in the direction normal to the interfaces between components. This is a well-known problem in the recent literature and we can mentioned here the papers by Hermann, Sun and others, [1-5] and Woźniak [6]. 
The aim of this contribution is to formulate two approaches to the long wave propagation problem by using the mass discretized approximation. It will be shown that these two approximations lead to completely different results.

\section{Object of analysis}

The object of subsequent analysis is the periodically stratified two-component linear elastic 3-D space. The period of the stratification will be denoted by $l$. A fragment of the 3-D space under consideration is shown in Figure 1.

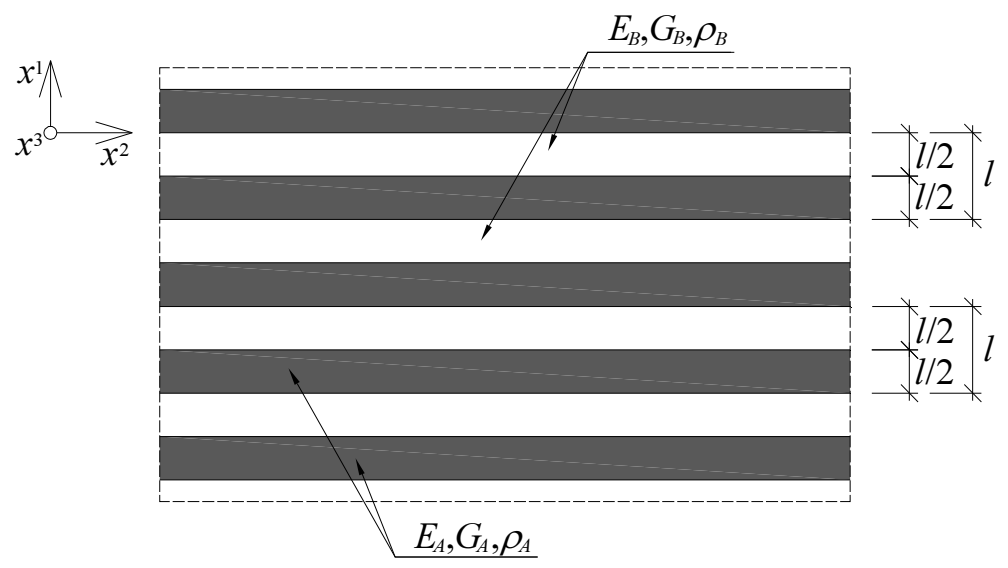

Fig. 1. A fragment of periodically stratified 3-D space; Symbols $E_{A}, G_{A}, \rho_{A}$ and $E_{B}$, $G_{B}, \rho_{B}$ stand for Young modulus, Kirchhoff modulus and mass density of constituents, respectively

Let $O x^{1} x^{2} x^{3}$ be the Cartesian orthogonal coordinate system in physical space, occupied by the elastic medium under consideration, where $O x^{1}$ - axis is normal to interfaces. The time coordinate will be denoted by $t, t \in R$. Moreover $x^{1}, x^{2}, x^{3}, t$ represent inertial coordinate in the space-time.

Material components will be specified by subscripts $A$ and $B$. Longitudinal Young and transversal Kirchhoff modulae of these components will be jointly denoted by $\left\{E_{A}, G_{A}\right\}$ and $\left\{E_{B}, G_{B}\right\}$, respectively. To simplify the formal manipulations it is assumed that $\rho_{A}=\rho_{B}$ and the mass density of both material components will be denoted by $\rho$.

For the waves propagating exclusively in the direction normal to interfaces we shall independently investigate the longitudinal and transversal vibrations and hence we denote $H_{A} \in\left\{E_{A}, G_{A}\right\}$ and $H_{B} \in\left\{E_{B}, G_{B}\right\}$. To simplify investigations it is assumed that the displacement field is independent of space coordinates $x^{2}, x^{3}$. 
We also obtain independent problems for displacement component $w^{1}(\cdot, t)$ in the direction $O x^{1}$ normal to the interfaces, as well as displacement components $w^{2}(\cdot, t), w^{3}(\cdot, t)$ in the directions $O x^{2}, O x^{3}$ mutually orthogonal and parallel to the interfaces. Hence we use denotations $w(\cdot, t) \in\left\{w^{1}(\cdot, t), w^{2}(\cdot, t), w^{3}(\cdot, t)\right\}$, where $w(\cdot, t)$ are assumed to be continuous up to the second space derivatives. Thus we shall deal with the 1-D dynamic model of the linear elastic medium under consideration.

\section{Mass discretization}

Under the formal condition $\rho_{A}=\rho_{B}=\rho$ the mass discretization of the 3-D elastic space can be performed on interfaces. The homogeneous layer bounded by $x_{1}=0, x_{1}=\frac{l}{2}$ will be uniformly occupied by material "A". Hence the homogeneous layer bounded by planes $x_{1}=-\frac{l}{2}, x_{1}=0$ is assumed to be occupied by material "B". The system of interfaces is given by $x^{1}=x_{n} \equiv n \frac{l}{2}, \quad n=0, \pm 1, \pm 2, \ldots$, (Fig. 1).

We shall analyze below two special cases of mass discretization. In the first case we assign to every interface $x^{1}=x_{n} \equiv n \frac{l}{2}$, the mass density $\rho \frac{l}{2}$. In the second case we assign mass density $\rho l$ exclusively to the even interfaces $x_{2 n}$, $n=0, \pm 1, \pm 2, \ldots$. This situation in which the mass is discretized on odd interfaces is similar as that assigned to the even interfaces.

2.1. Mass discretized on interfaces $x^{1}=x_{n} \equiv n \frac{l}{2} n=0, \pm 1, \pm 2, \ldots$

In this case the dynamic equilibrium conditions are:

$$
\begin{aligned}
& H_{B}\left(w_{2 n+1}(t)-w_{2 n}(t)\right)-H_{A}\left(w_{2 n}(t)-w_{2 n-1}(t)\right)=\frac{l}{4} \rho \ddot{w}_{2 n}(t), \\
& H_{A}\left(w_{2 n+2}(t)-w_{2 n+1}(t)\right)-H_{B}\left(w_{2 n+1}(t)-w_{2 n}(t)\right)=\frac{l}{4} \rho \ddot{w}_{2 n+1}(t) .
\end{aligned}
$$

where here and subsequently $w_{n}(\cdot, t)$ are displacements for $x^{1}=x_{n}, n=0, \pm 1, \pm 2, \ldots$. 
In this Subsection we shall confine ourselves to the harmonic waves propagating in the direction of the $O x^{1}$-axis. Let $\lambda$ stand for the wavelength, $\kappa \equiv \frac{2 \pi}{\lambda}$ the wave number, $k \equiv \frac{2 \pi l}{\lambda}=\kappa l$ the dimensionless wave number, and by $\omega$ free vibration frequency in 3-D linear elastic space.

To this end we shall look for harmonic vibrations in the form

$$
\begin{aligned}
& w_{2 n}(t)=C_{1} \exp i(\omega t-n k), \\
& w_{2 n+1}(t)=C_{2} \exp i\left[\omega t-(2 n+1) \frac{k}{2}\right], \quad n=0, \pm 1, \pm 2, \ldots
\end{aligned}
$$

where $C_{1}, C_{2}$ are arbitrary constants.

Substituting the right-hand sides of the above formulas into (1) we obtain the homogenous system of linear algebraic equations for $C_{1}, C_{2}$. By applying the procedure similar to that given in [5] Chapter IV we obtain the dispersion relation between $\omega$ and $k$ in the form given by two branches:

$$
\begin{aligned}
& \omega_{-}^{2}=\frac{8\left(H_{A}+H_{B}\right)}{l^{2} \rho} \sin ^{2} \frac{k}{4}, \\
& \omega_{+}^{2}=\frac{8\left(H_{A}+H_{B}\right)}{l^{2} \rho} \cos ^{2} \frac{k}{4} .
\end{aligned}
$$

Under denotation:

$$
a^{2} \equiv \frac{8}{l^{2} \rho}\left(H_{A}+H_{B}\right)
$$

the diagram of dispersion relation (3) is shown in Figure 2.

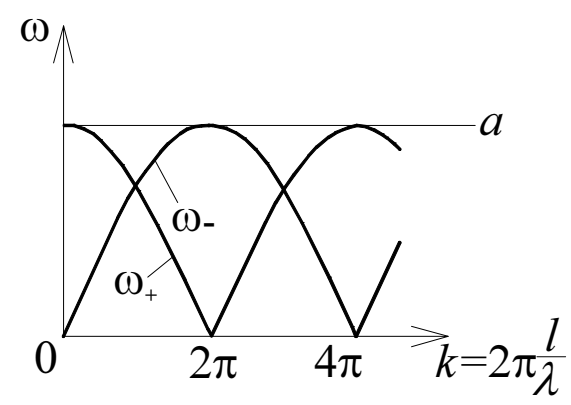

Fig. 2. Diagram of dispersion relation 
It can be seen that there exist two independent branches of the dispersion curves which are mutually intersecting. However, it has to be remembered that from the physical point of view we shall take into account only the part of the dispersion relation for long wave i.e. for $0<k<<1$.

Let us apply formulas (3) to free vibrations which hold only for long waves i.e. for $0<k<<1$. In this situation formulas (3) can be rewritten in the form:

$$
\begin{aligned}
& \omega_{-}^{2}=\frac{\left(H_{A}+H_{B}\right)}{2 \rho} \kappa^{2}+O\left(k^{2}\right), \\
& \omega_{+}^{2}=\frac{8\left(H_{A}+H_{B}\right)}{l^{2} \rho}+\frac{H_{A}+H_{B}}{2 l^{2} \rho} k^{2}+O\left(k^{2}\right),
\end{aligned}
$$

For an arbitrary positive $\lambda$ and under limit passage $k \rightarrow 0$ we obtain:

$$
\begin{aligned}
& \omega_{-}^{2} \rightarrow \frac{\left(H_{A}+H_{B}\right)}{2 \rho} \kappa^{2}, \\
& \omega_{+}^{2} \rightarrow \infty .
\end{aligned}
$$

we recall that $\kappa \equiv \frac{2 \pi}{\lambda}$. The physical meaning of this result will be discussed in Conclusions.

\subsection{Mass discretized on interfaces $x^{1}=x_{n} \equiv n l, n=0, \pm 1, \pm 2, \ldots$}

In this case the dynamic equilibrium conditions are:

$$
\begin{aligned}
& H_{B}\left(w_{2 n+1}-w_{2 n}\right)-H_{A}\left(w_{2 n}-w_{2 n-1}\right)=\frac{l^{2} \rho}{2} \ddot{w}_{2 n}, \\
& H_{A}\left(w_{2 n+2}-w_{2 n+1}\right)-H_{B}\left(w_{2 n+1}-w_{2 n}\right)=0
\end{aligned}
$$

Obviously the second from the above conditions represents the equilibrium on interfaces deprived mass distribution, $x^{1}=n \frac{l}{2}, n=0, \pm 1, \pm 2, \ldots$

From the second of the above conditions we obtain $w_{2 n+1}$ in the terms of $w_{2 n}$ and $w_{2 n+2}$. Substituting this result into first equation from (7) we arrive at the final result in the form:

$$
\frac{2 H_{A} H_{B}}{H_{A}+H_{B}}\left(w_{2 n+2}-2 w_{2 n}+w_{2 n-2}\right)=l^{2} \rho \ddot{w}_{2 n}, \quad n=0, \pm 1, \pm 2, \ldots
$$

This is the main result of the mass discretization on interfaces $x^{1}=x_{n} \equiv n l$. 
Denoting by:

$$
\begin{gathered}
H_{0} \equiv \frac{2 H_{A} H_{B}}{H_{A}+H_{B}}, \\
\Delta w_{2 n+1} \equiv \frac{w_{2 n+2}-w_{2 n}}{l}, \\
\Delta w_{2 n-1} \equiv \frac{w_{2 n}-w_{2 n-2}}{l}, \\
\Delta \Delta w_{2 n} \equiv \frac{\Delta w_{2 n+1}-w_{2 n-1}}{l},
\end{gathered}
$$

we can rewrite (8) in the finite-difference form:

$$
H_{0} \Delta \Delta w_{2 n}=\rho \ddot{w}_{2 n}, n=0, \pm 1, \pm 2, \ldots
$$

From (9) and for $l \rightarrow 0$ we shall derive what will be called the asymptotic form of the long wave approximation of (9). To this end let $u\left(x^{1}, t\right), x^{1} \in R, t \in R$ $u(\cdot, t) \in\left\{u^{1}(\cdot, t), u^{2}(\cdot, t), u^{3}(\cdot, t)\right\}$ satisfy condition $u(\cdot, t) \in C^{2}((0, L))$, where $\mathrm{L}>>1$ for an arbitrary but fixed interval $[0, L]$ of $O x^{1}$ axis. The resulting asymptotic form of the long wave approximation for $l \rightarrow 0$ yields:

$$
H_{0} \partial^{2} u-\rho \ddot{u}=0
$$

This means that under the limit passage $l \rightarrow 0$ we obtain the well-known wave equation representing the asymptotic model of 1-D elastodynamics in the layer $(0, L) \times R^{2}$.

At the end of this subsection we pass to the free vibration problem of the mass discretized space under consideration. Similarly like in Subsection 2.1 we shall look for the solution to Equation (8) in the form:

$$
w_{2 n}(t)=C \exp i(\omega t-n k), \quad n=0, \pm 1, \pm 2, \ldots
$$

Substituting the right hand side of the above solution into Equation (9) after some manipulations we obtain dispersion relation:

$$
\omega^{2}=\frac{4 H_{0}}{\rho l^{2}} \sin ^{2} \frac{k}{2}
$$

Under denotation:

$$
b^{2} \equiv \frac{4 H_{0}}{l^{2} \rho}
$$


the diagram of the dispersion relation is shown in Figure 3.

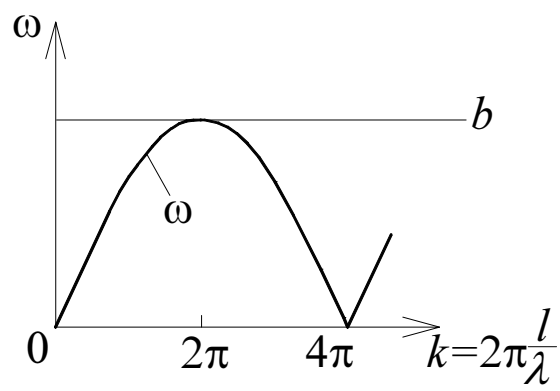

Fig. 3. Diagram of dispersion relation

In this case the dispersion relation has only one branch.

Applying formula (11) to the free vibration which hold only for long wave i.e. for $0<k<<1$ the formula (12) can be rewritten in the form

$$
\omega^{2}=\frac{H_{0}}{\rho} \kappa^{2}+O\left(k^{2}\right)
$$

For an arbitrary positive $\lambda$ and under limit passage $k \rightarrow 0$ we obtain:

$$
\omega^{2}=\frac{H_{0}}{\rho} \kappa^{2}
$$

we recall that $\kappa \equiv \frac{2 \pi}{\lambda}$. This result corresponds to the well-known results.

\section{Conclusions}

The main concluding remark is that the free vibration frequency in Case 1 tends either to $\frac{\left(H_{A}+H_{B}\right)}{2 \rho} \kappa^{2}$ or to infinity. However, the result given by formula (8) from the physical point of view is completely wrong in any case in which $H_{A} \neq H_{B}$. It can be easily seen that for $H_{A}=0$ or $H_{B}=0$ (but not both!) in the framework of Case 1 the frequency of free vibrations $\omega_{-}^{2}$ is positive and finite, for every $\lambda>0$.

Hence the final conclusion is that the proposed mass discretization approach, given here as the Case 1 cannot be treated as correct.

Obviously for the wave propagating in the direction of the $O x^{1}$-axis vibration frequencies (5) for $H_{A}=0$ and $H_{B}>0$ or $H_{A}>0$ and $H_{B}=0$ have to be equal to zero which is in contradiction to formula (5). On the other hand all results pre- 
sented in Subsection (2.2) have a physical sense provided that the length wave $\lambda$ is sufficiently large when compared to inhomogenity period $l$.

The proposed discretization which is presented as Case 2 leads to asymptotic model which is physically permissible.

\section{References}

[1] Sun C.T., Achenbach J.C., Herrmann G., Time-harmonic waves in a stratified medium propagating in the direction of the layering, J. Appl. Mech. 1968, 35, 408.

[2] Delph T.J., Herrmann G., Kaul R.K., Harmonic wave propagation in a periodically layered, infinite elastic body: Antiplane strain, J. Appl. Mech. 1978, 45, 343.

[3] Delph T.J., Herrmann G., Kaul R.K., Harmonic wave propagation in a periodically layered, infinite elastic body: Plane strain, Analytical results, J. Appl. Mech. 1979, 46, 113.

[4] Delph T.J., Herrmann G., Kaul R.K., Harmonic Wave propagation in a periodically layered, infinite elastic body: Plane strain, Numerical results, J. Appl. Mech. 1980, 47, 531.

[5] Brillouin L., Wave Propagation in Periodic Structures, McGraw-Hill Company 1946.

[6] Woźniak C., Internal variables in dynamics of composites solids with periodic microstructure, Arch. Mech. 1997, 49, 421-441. 